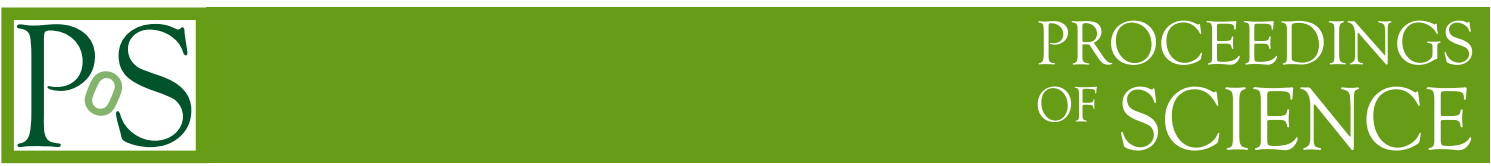

\title{
Search for leptoquarks using the ATLAS detector
}

\section{Yasuyuki Okumura*}

on behalf of the ATLAS collaboration, International Center for Elementary Particle Physics, The University of Tokyo

E-mail: yasuyuki.okumura@cern.ch

Searches for pair production of scalar leptoquarks performed by the ATLAS experiment at Large Hadron Collider are presented. The full Run-2 dataset of $p p$ collisions recorded with the ATLAS detector is exploited, corresponding to an integrated luminosity of $139 \mathrm{fb}^{-1}$. Three classes of final states are considered: an oppositely-charged same-flavour light-lepton pair (either two electrons or two muons) and two jets to search for leptoquarks decaying into a jet and a light-lepton; light-leptons and at least one hadronically decaying $\tau$-lepton, which is optimised for a search for leptoquarks decaying into a top-quark and a $\tau$-lepton; an oppositely-charged same-flavour lightlepton pair and two large-radius jets as from decay products of boosted top quarks for leptoquarks decaying into a top-quark and a light-lepton. Various leptoquark models resulting in different choices of quark- and lepton-generations in the decays are tested. No significant excesses above the Standard Model expectations are observed in any of the considered final states, and 95\% CL upper limits are set on the production cross-section as a function of the leptoquark mass and the branching fraction, where the branching fraction is considered between two decay modes with charged-leptons and neutrinos for given configurations of quark- and lepton-generations. Under the assumption of the branching fraction into a charged lepton and a quark of $100 \%$, leptoquarks with masses below 1.4-1.8 TeV are excluded, depending on the leptoquark models.

40th International Conference on High Energy physics - ICHEP2020

July 28 - August 6, 2020

Prague, Czech Republic (virtual meeting)

${ }^{*}$ Speaker 


\section{Introduction}

Many extensions of the Standard Model of the particle physics (SM) contain leptoquarks (LQs), motivated by the known similarities in the structures of the quark and lepton sectors. LQs are hypothetical new particles that carry non-zero baryon and lepton numbers. Because of their quantum numbers, a Yukawa interaction allows scalar LQs to couple to a pair of a lepton and a quark at an interaction vertex with coupling constants that can vary for different combinations of generations of lepton-quark pairs, controlled by model parameters. The LQs will carry the colour- and electriccharges, satisfying the gauge symmetry in the interaction. Consequently, intermediate scalar LQs allow processes violating lepton flavour universality, and therefore, possibly explain recent measurements of $B$-meson decays that show deviations from SM predictions. As the scalar LQs are triplets for the strong interaction, in high energy $p p$ collisions, they are supposed to be produced mainly in pairs with gluon-gluon fusion and quark-antiquark annihilation via the strong interaction. The LQs decay into a pair of a lepton and a quark, according to the considered LQ models. The Buchmüller-Rückl-Wyler model describes leptoquark phenomenology in an assumption that LQs can only interact with leptons and quarks of the same generation such as $\mathrm{LQ}_{3}^{\mathrm{d}} \rightarrow t \tau$, where $\mathrm{LQ}_{3}^{\mathrm{d}}$ stands for a LQ decaying into third-generation leptons and quarks with an electric charge of $\pm 1 / 3 e$. Besides, recent LQ searches extend the models so that cross-generational LQ decays are considered such as LQ $\rightarrow t e$ or LQ $\rightarrow t \mu$. Two parameters model a Yukawa interaction to a given quarkgeneration and a lepton-generation: model parameters $\beta$ and $\lambda$. Consequently, the coupling to the charged lepton is given by $\sqrt{\beta} \lambda$, while the coupling to the neutrino is given by $\sqrt{1-\beta} \lambda$, where the $\beta$ parameter determines the branching fraction $B$ between charged-lepton and neutrino modes for given configurations of quark- and lepton-generations involved in the Yukawa interactions.

This paper presents three searches for pair production of scalar LQs performed by the ATLAS collaboration, which exploit the full Run $2 p p$ collisions dataset at $\sqrt{s}=13 \mathrm{TeV}$ recorded with the ATLAS detector at the Large Hadron Collider (LHC). The corresponding integrated luminosity is $139 \mathrm{fb}^{-1}$. Three classes of final states are considered to test various models of the LQs: an oppositely-charged same-flavour light-lepton pair (either two electrons or two muons) and two jets; one light lepton and at least one hadronically decaying $\tau$-lepton; an oppositely-charged same-flavour light-lepton pair and two large-radius jets originated from decay products of boosted top quarks.

The ATLAS detector [1] covers nearly the entire solid angle around the interaction point. It consists of tracking detectors surrounded by a thin superconducting solenoid magnet, electromagnetic and hadronic calorimeters, and external muon spectrometers incorporating a large superconducting toroidal magnet system. A two-level trigger system provides online selection in data acquisition. Electron candidates are reconstructed from energy clusters in the electromagnetic calorimeter that are associated with inner detector tracks. Muon candidates are reconstructed by matching tracks found in the muon spectrometers and the inner tracking detectors. Hadronically decaying $\tau$-lepton candidates $\left(\tau_{\text {had }}\right)$ are reconstructed from clusters in the calorimeters and associated inner detector tracks, requiring either one or three associated tracks with a total charge of $\pm 1 e$. Jets with a distance parameter $R=0.4$ are reconstructed by combining measurements from both the tracker and the calorimeter using the particle flow algorithm. Jets with $R=1.0$ (denoted as large-R jets) are reconstructed based on the calorimeter clusters. The anti- $k_{t}$ algorithm is used for both types of jets. Jets are categorised into $b$-tagged, $c$-tagged, and light-flavour jets with an algorithm that exploits 
multivariate techniques to combine information about the impact parameters of displaced tracks and the topological properties of secondary and tertiary decay vertices reconstructed within the jet. The missing transverse energy, $E_{\mathrm{T}}^{\mathrm{miss}}$, is calculated as the negative vectorial sum of the transverse momenta of all fully calibrated hard objects and soft contributions calculated with charged tracks associated with the most energetic primary vertex of the events.

\section{Search for pairs of scalar leptoquarks decaying into quarks and electrons or muons}

A search for scalar leptoquarks that decay into a light-lepton $(\ell)$ and a jet $(j)$ performed by the ATLAS experiment has been performed [2]. The search is performed in a final state characterised by two electrons or two muons and two or more jets for either LQ $\overline{L Q} \rightarrow e j e j$ or $\mu j \mu j$. LQ candidates can be reconstructed as a lepton-jet pair, where the solution of pairing is found by selecting a combination that makes the two invariant masses of the lepton-jet systems of the candidate events closest. A tagging algorithm is used to identify jets originating from the fragmentation of $b$ quarks, $c$-quarks, and the other light-flavour quarks $(u, d, s$; denoted as $q)$. Analyses are optimised separately for six classes of scalar LQ models that are compatible with the considered final states: LQ $\rightarrow q / c / b+e$ and LQ $\rightarrow q / c / b+\mu$. Especially, searches for LQs decaying into either $b$-quarks or $c$-quarks exploit event categorisation based on the flavours to maximise the search sensitivity. A maximum-likelihood fit is performed on the distributions of the average mass of the LQ candidates $\left(m_{\ell j}^{\mathrm{Av}}\right)$ to extract the LQ signals. Figures 1 (a) and (b) show the post-fit $m_{\ell j}^{\mathrm{Av}}$ distributions observed in data categories to search for $\mathrm{LQ} \rightarrow q e$ and $\mathrm{LQ} \rightarrow q \mu$ signals, respectively. No significant excess above the SM expectation is observed on the $m_{\ell j}^{\mathrm{Av}}$ spectrum for all six classes of the LQ models, and 95\% CL upper limits are set on the production cross-section as a function of the LQ mass under different assumptions of the branching fractions into charged lepton and neutrino for the six models, individually. LQs with masses below $1.8 \mathrm{TeV}$ and $1.7 \mathrm{TeV}$ are excluded in the electron and muon channels, respectively, assuming a $100 \%$ branching fraction into a charged lepton and a quark, for all the quark flavour scenarios. Figure 2 shows the branching fraction upper limit for LQ $\rightarrow q e$ and $\mathrm{LQ} \rightarrow q \mu$ signals in a mass range between $400 \mathrm{GeV}$ and $2 \mathrm{TeV}$.

\section{Search for pair production of third-generation scalar leptoquarks decaying into a top quark and a $\tau$-lepton}

A search for scalar leptoquarks decaying into a top quark and a $\tau$-lepton has been performed [3], where LQs decaying into third-generation leptons and quarks with an electric charge of $\pm 1 / 3 e$ are denoted as $\mathrm{LQ}_{3}^{\mathrm{d}}$. The $\mathrm{LQ}_{3}^{\mathrm{d}} \overline{\mathrm{LQ}_{3}^{\mathrm{d}}} \rightarrow t \tau t \tau$ signal events often decay into a final state that contains at least one light-lepton from a semileptonic top-quark decay or a leptonic $\tau$-lepton decay, which can be used for triggering the events. Requirements on the additional $\tau_{\text {had }}$ candidates provide further reduction of the SM backgrounds and improve the search sensitivity. Therefore, the final state of interest is characterised by light-leptons, at least one $\tau_{\text {had }}$, and additional jets. Event categorisation into signal, validation, and control regions are optimised to search for the signal and improve the modelling of leading backgrounds simultaneously with a maximum-likelihood fit. The most sensitive event categories require at least one $\tau_{\text {had }}$ candidate and exploit the effective mass 


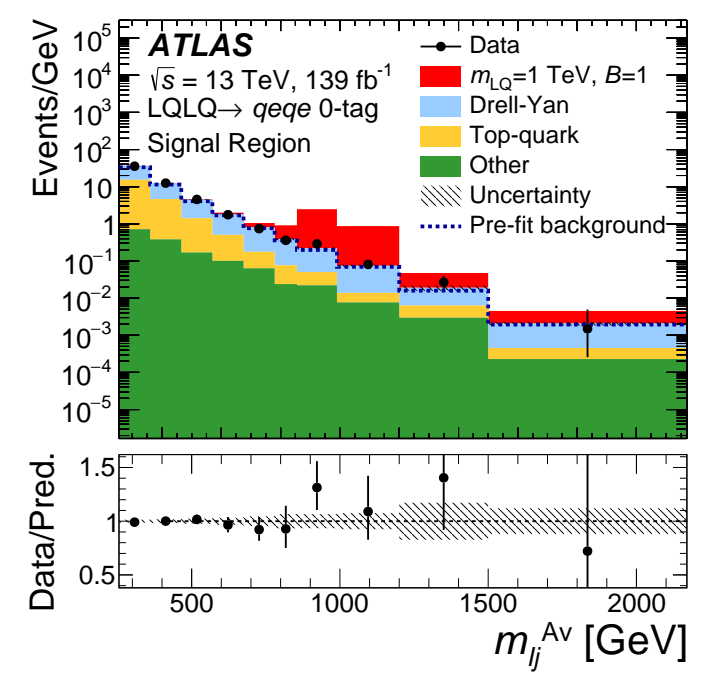

(a)

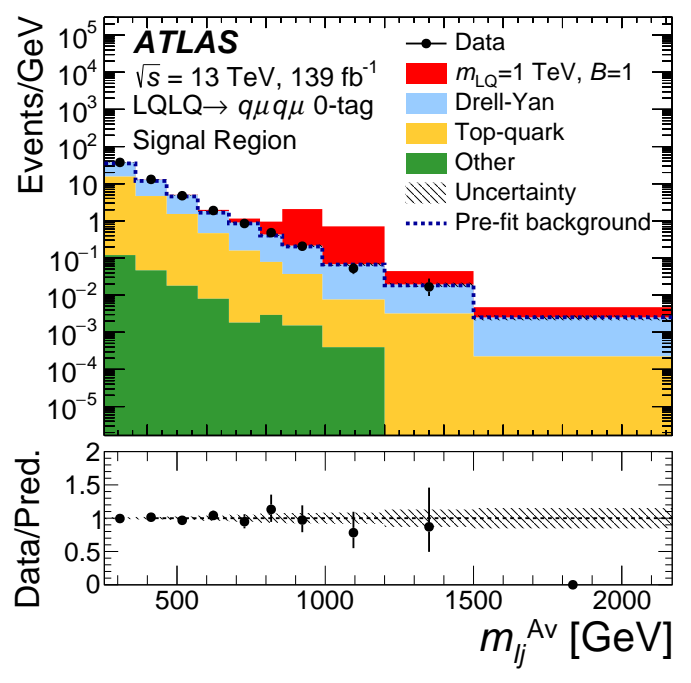

(b)

Figure 1: Post-fit distributions of $m_{\ell j}^{\mathrm{A} v}$ in the signal regions for (a) LQ $\rightarrow q e$ and (b) LQ $\rightarrow q \mu$ signal searches, where the $q$ stands for light-flavour quarks $(u, d, s)$. For LQs decaying into light-flavour quarks. The expected signals are shown for $m_{\mathrm{LQ}}=1 \mathrm{TeV}$ for reference [2].

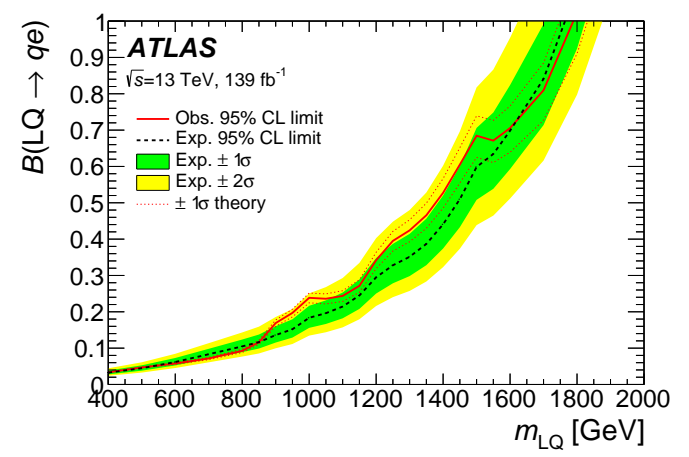

(a)

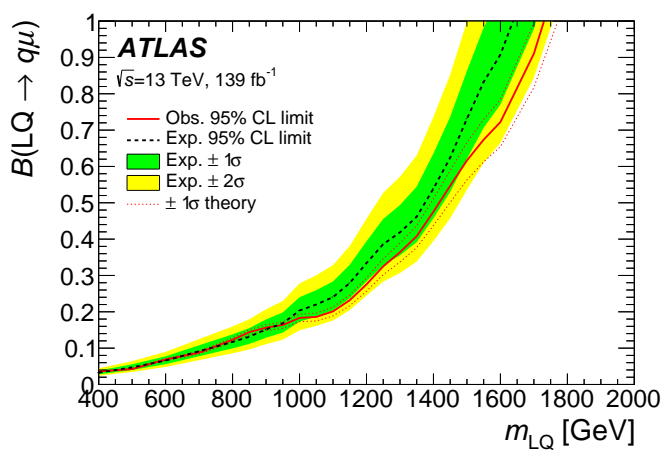

(b)

Figure 2: The observed and expected limits on the LQ branching fraction at 95\% CL as a function of $m_{\mathrm{LQ}}$ for (a) LQ $\rightarrow q e$ and (b) LQ $\rightarrow q \mu$ signals, where $q$ stands for light-flavour quarks $(u, d, s)$, and the limits are applicable for all the three quark flavours [2].

distributions, which is defined as the scalar sum of the transverse momentum of the selected jets and leptons, and the $E_{\mathrm{T}}^{\text {miss }}$, as the final discriminant to discriminate between the signal and the background. The modelling of leading backgrounds of $t \bar{t}$ is improved with a kinematic correction, and the modelling of reducible backgrounds due to the hadron jets mimicking $\tau_{\text {had }}$ is improved by a data-driven correction factor of the background rate. Owing to the precise estimation of the backgrounds and optimal selections, the search sensitivity reaches an expected significance of five standard deviations for a scalar LQ decaying with $m_{\mathrm{LQ}_{3}^{\mathrm{d}}}=1.2 \mathrm{TeV}(960 \mathrm{GeV})$ under the assumption of $B\left(\mathrm{LQ}_{3}^{\mathrm{d}} \rightarrow t \tau\right)=1.0(0.5)$, while the observed data are compatible with the SM expectation 
(see Figure 3 (a)). 95\% CL upper limits are set on the production cross-section as a function of $m_{\mathrm{LQ}_{3}^{\mathrm{d}}}$ under various assumptions of branching fractions into $t \tau$ and $b v_{\tau}$. The $\mathrm{LQ}_{3}^{\mathrm{d}}$ is excluded up to $1.43 \mathrm{TeV}$ for the $B\left(\mathrm{LQ}_{3}^{\mathrm{d}} \rightarrow t \tau\right)=1.0$ assumption.

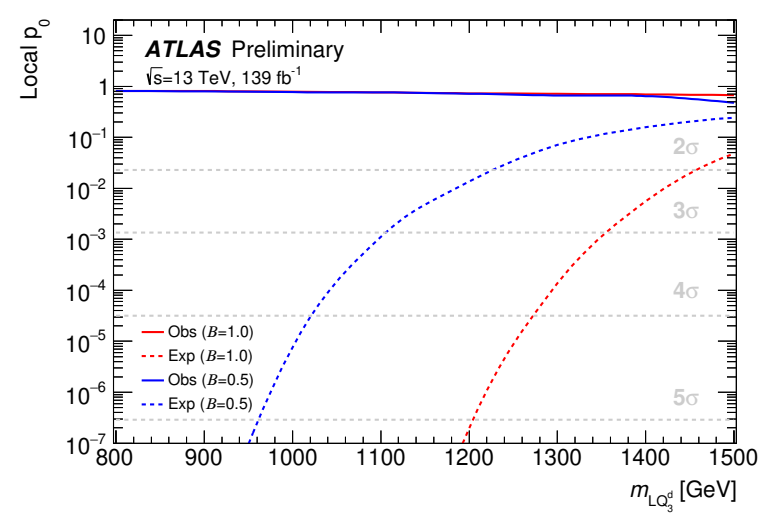

(a)

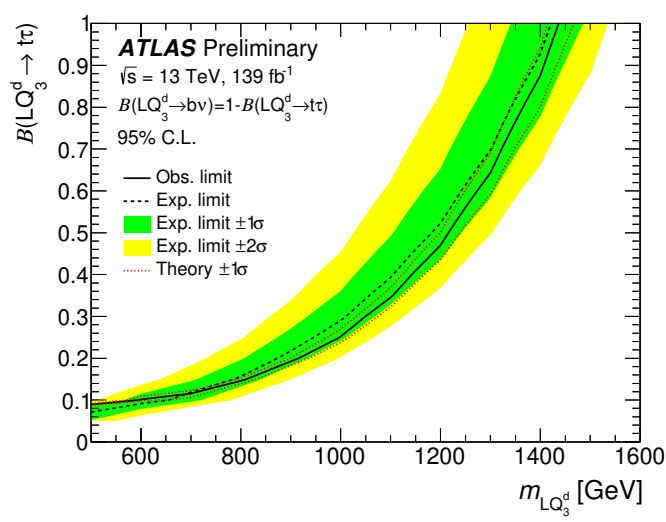

(b)

Figure 3: (a) The observed (solid) local $p_{0}$ as a function of $m_{\mathrm{LQ}_{3}^{\mathrm{d}}}$ for the assumptions of $B\left(\mathrm{LQ}_{3}^{\mathrm{d}} \rightarrow t \tau\right)=0.5$ (blue) and $B\left(\mathrm{LQ}_{3}^{\mathrm{d}} \rightarrow t \tau\right)=1.0$ (red). The dashed curve shows the expected local $p_{0}$ under the assumption of the presence of signals with the given $m_{\mathrm{LQ}_{3}^{\mathrm{d}}}$ and $B\left(\mathrm{LQ}_{3}^{\mathrm{d}} \rightarrow \tau \tau\right)$. (b) Observed (solid line) and expected (dashed line) $95 \% \mathrm{CL}$ upper limits on $B\left(\mathrm{LQ}_{3}^{\mathrm{d}} \rightarrow t \tau\right)$ as a function of $m_{\mathrm{LQ}_{3}^{\mathrm{d}}}$ [3].

\section{Search for pair production of scalar leptoquarks decaying into first- or second-generation leptons and top quarks}

A search for pair production of scalar leptoquarks decaying into a top quark and either an electron or a muon (LQ $\rightarrow t e$ and LQ $\rightarrow t \mu$ ) has been performed [4]. Candidate events are characterised by two large- $\mathrm{R}$ jets that are supposed to contain all decay products of top quarks associated with either two electrons or two muons so that the search sensitivity is maximised for high mass scalar LQs in the analysis. A Boosted Decision Tree (BDT) classifier is built to discriminate the LQ signals from the leading $t \bar{t}$ and $Z+$ jets backgrounds (see Figure 4 (a) for the electron channel). A maximum likelihood fit on the output score of the BDT classifier is performed to simultaneously determine the normalisation of the leading $t \bar{t}$ and $Z+$ jets backgrounds and search for the LQ signals. No significant excess above the SM expectation is observed and 95\% CL upper limits are set on the production cross-section as a function of $m_{\mathrm{LQ}}$ under various assumptions of branching fractions into $t \ell$ and $b v_{\ell}$ in both the electron and muon channels. The LQ models are excluded up to $1.5 \mathrm{TeV}$ for both electron and muon channels, under the assumption of $B(\mathrm{LQ} \rightarrow t \ell)=1.0$ (see Figure 4 (b) for the electron channel).

\section{Conclusion}

The ATLAS collaboration has been conducting searches for pair production of scalar leptoquarks, using a dataset of $p p$ collisions at $\sqrt{s}=13 \mathrm{TeV}$ recorded with the ATLAS detector during 


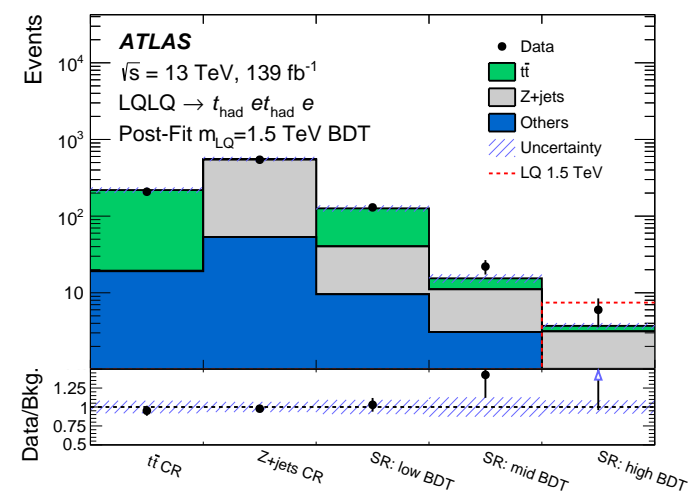

(a)

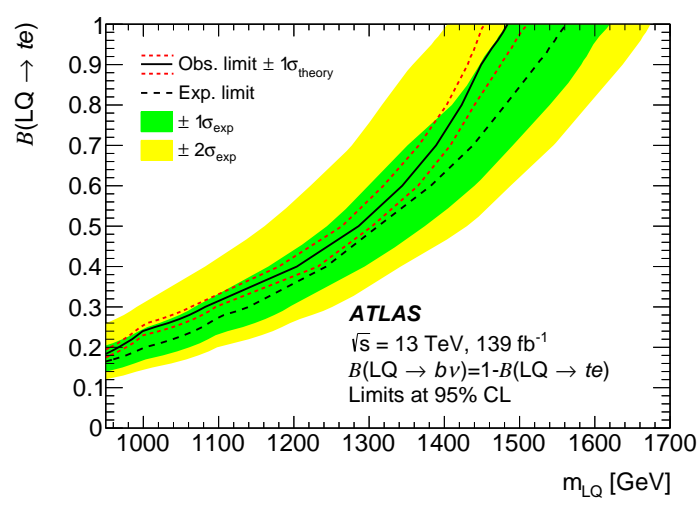

(b)

Figure 4: (a) Post-fit distributions of the BDT output score for LQ $\rightarrow$ te signals with a background-only hypothesis. The expected signals are shown for $m_{\mathrm{LQ}}=1.5 \mathrm{TeV}$ for reference (red dashed line). (b) Observed (solid line) and expected (dashed line) $95 \% \mathrm{CL}$ upper limits on $B\left(\mathrm{LQ} \rightarrow t e\right.$ ) as a function of $m_{\mathrm{LQ}}$ [4].

the Run 2 of the LHC, corresponding to an integrated luminosity of $139 \mathrm{fb}^{-1}$. Three final states are considered for testing various LQ models: LQs decaying into a jet and a light-lepton; LQs decaying into a top-quark and a $\tau$-lepton; LQs decaying into a top-quark and a light-lepton. No significant excesses above the SM expectations are observed in any of considered final states, and 95\% CL upper limits are set on the production cross-section as a function of the LQ mass and branching fraction, where the branching fraction is considered between two decay modes with charged-leptons and neutrinos for the given configurations of quark- and lepton-generations involved in the LQ decays. The analysis optimised for LQ $\rightarrow e j$ and $\mu j$ signals excludes LQs with masses below $1.8 \mathrm{TeV}$ and $1.7 \mathrm{TeV}$ in the electron and muon channels, respectively. The limits are valid for different quark flavors with a minimal dependency under the assumption of a $100 \%$ branching fraction into a charged light-lepton and a quark. The third-generation scalar LQ $\left(\mathrm{LQ}_{3}^{\mathrm{d}}\right)$ models are excluded up to $1.43 \mathrm{TeV}$ for the assumption of $B\left(\mathrm{LQ}_{3}^{\mathrm{d}} \rightarrow t \tau\right)=1.0$, by a dedicated analysis in a final state characterised by light-leptons and at least one hadronically decaying $\tau$-lepton. The search with light-leptons and large-R jets excludes the LQ models up to $1.5 \mathrm{TeV}$ for both $\mathrm{LQ} \rightarrow t e$ and $t \mu$ models under the assumption of $B(\mathrm{LQ} \rightarrow t \ell)=1.0$.

\section{References}

[1] ATLAS Collaboration, JINST 3 (2008) S08003.

[2] ATLAS Collaboration, JHEP 10 (2020) 112.

[3] ATLAS Collaboration, ATLAS-CONF-2020-029, http://cdsweb.cern.ch/record/ 2725735.

[4] ATLAS Collaboration, arXiv:2010.02098 [hep-ex] (submitted to EPJC). 